\title{
FINDING COMPOUND STRUCTURES IN IMAGES USING IMAGE SEGMENTATION AND GRAPH-BASED KNOWLEDGE DISCOVERY
}

\author{
Daniya Zamalieva, Selim Aksoy \\ Department of Computer Engineering \\ Bilkent University \\ Bilkent, 06800, Ankara, Turkey \\ \{daniya,saksoy\}@cs.bilkent.edu.tr
}

\author{
James C. Tilton \\ Computational \& Information Sciences \\ and Technology Office (606.3) \\ NASA Goddard Space Flight Center \\ Greenbelt, MD 20771, USA \\ James.C.Tilton@nasa.gov
}

\begin{abstract}
We present an unsupervised method for discovering compound image structures that are comprised of simpler primitive objects. An initial segmentation step produces image regions with homogeneous spectral content. Then, the segmentation is translated into a relational graph structure whose nodes correspond to the regions and the edges represent the relationships between these regions. We assume that the region objects that appear together frequently can be considered as strongly related. This relation is modeled using the transition frequencies between neighboring regions, and the significant relations are found as the modes of a probability distribution estimated using the features of these transitions. Experiments using an Ikonos image show that subgraphs found within the graph representing the whole image correspond to parts of different high-level compound structures.
\end{abstract}

Index Terms - Image segmentation, object detection, graph-based analysis

\section{INTRODUCTION}

The common goal of object-based image analysis techniques in the literature is to partition the images into homogeneous regions and classify these regions. However, such homogeneous regions often correspond to very small details in very high spatial resolution images obtained from the new generation sensors. One interesting way of enabling the highlevel understanding of the image content is to identify the image regions that are intrinsically heterogeneous. These image regions are comprised of primitive objects of many diverse types, and can also be referred to as compound objects.

In comparison to the single object detection, the studies that aim to detect the compound objects are not encountered frequently in the literature. In one attempt for detecting compound objects of predefined types, Bhagavathy and Manjunath [1] build a texture motif model for harbors and golf

Daniya Zamalieva and Selim Aksoy were supported in part by the TUBITAK CAREER grant 104E074. courses from training examples. Dogrusoz and Aksoy [2] detect organized and unorganized urban areas by clustering a scene graph whose nodes correspond to individual buildings. Stasolla and Gamba [3] detect built-up areas in highresolution SAR images using local autocorrelation.

In this paper, we propose a generic unsupervised method for discovering interesting and significant compound objects regardless of their types. The method translates image segmentation into a relational graph, and applies a graph-based knowledge discovery algorithm to find the interesting and repeating substructures that may correspond to compound objects. The first step is image segmentation where the resulting regions correspond to primitive objects that have relatively uniform spectral content (Section 2). The next step is the translation of this segmentation into a relational graph structure where the nodes represent the regions and the edges represent the relationships between these regions. We assume that the region objects that appear together frequently can be considered as strongly related. This relation is modeled using the transition frequencies between neighboring regions. Each transition is represented by a point in a multi-dimensional space. This space is modeled by a non-parametric probability distribution, and the local maxima found from the density function are assumed to correspond to the most frequently occurring and hence the most significant and important transitions (Section 3). Finally, a graph whose edges encode this frequent spatial co-occurrence information is constructed, and a subgraph analysis algorithm is used to discover substructures that often correspond to groups of region objects that occur together in high-level compound structures (Section 4). Proof-of-concept experiments illustrate the proposed algorithm on an Ikonos image (Section 5).

\section{SEGMENTATION AND FEATURE EXTRACTION}

The first step is image segmentation where the regions found correspond to primitive objects that have relatively uniform spectral content. We use the Recursive Hierarchical Segmentation (RHSEG) algorithm [4] for this segmentation step. 


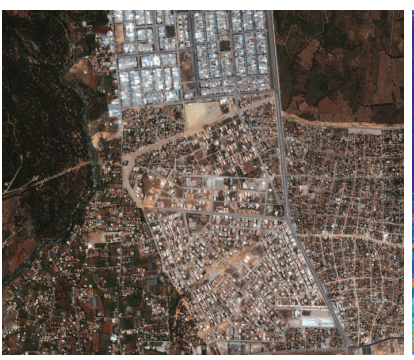

(a) Ikonos image

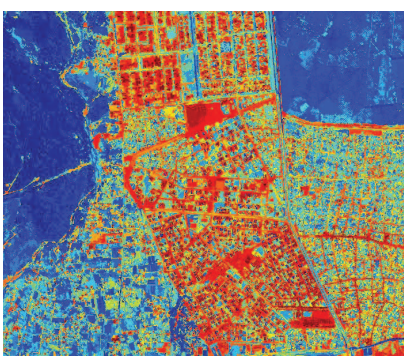

(b) Segmentation
Fig. 1. An Ikonos image of Antalya, Turkey and its segmentation.

RHSEG is a promising choice because of three key factors: (i) the high spatial fidelity of image segmentations produced by RHSEG, (ii) automatic grouping of the spatially connected region objects into region classes, and (iii) automatic production of a hierarchical set of segmentations. It is possible to examine how the regions change at each level and choose the level of detail at which the particular regions of interest are delineated. Figure 1 shows a multi-spectral Ikonos image of Antalya, Turkey with $4 \mathrm{~m}$ spatial resolution and $700 \times 600$ pixel size, along with its segmentation in false color.

The regions obtained from segmentation are represented using their spectral and size information. The spectral features for each region are computed using the average red, green and blue values of the pixels in that region. The size information corresponds to the number of pixels in each region. We use size as a feature to be able to distinguish regions with similar spectral content but significantly different sizes. All features are normalized to the $[0,1]$ range using linear scaling. Finally, each region $R_{i}$ is represented using the feature vector $\mathbf{y}_{\boldsymbol{i}}=\left(r_{i}, g_{i}, b_{i}, s_{i}\right)$ with 4 components.

\section{MODELING REGION CO-OCCURRENCE}

The next step is the translation of this segmentation into a relational graph structure where the nodes correspond to the individual regions, and the edges model their spatial relationships. In this paper, we model the region relationships using the transition frequencies between neighboring regions in the image by assuming that the region objects that appear together frequently in the image can be considered as strongly related.

One way to calculate the inter-region transition frequency is by determining the types of the regions and by counting the transitions involving the same types of region pairs. However, the determination of region types is a challenging classification problem, and errors at this step will result in misleading transition types. We propose to use a spatial co-occurrence model that enables transition frequency calculation without a preceding transition or region type assignment. This model involves a multi-dimensional space where each point corresponds to an inter-region transition, and enables the incorpo- ration of region transition frequencies together with region features. The space is modeled by a non-parametric probability distribution so that the probability value for each transition point corresponds to the frequency of its occurrence in the image. The details of this model are described below.

\subsection{Spatial co-occurrence space}

Each inter-region transition is defined by the features of the corresponding regions so that their contents can be incorporated in the model. In an image with $N_{R}$ regions $R_{i}, i=$ $1, \ldots, N_{R}$, the transition $T_{i j}$ involving the regions $R_{i}$ and $R_{j}$ is represented by the concatenation of the feature vectors of the two regions as $\mathbf{y}_{\boldsymbol{i} \boldsymbol{j}}=\left(\mathbf{y}_{\boldsymbol{i}}, \mathbf{y}_{\boldsymbol{j}}\right)$. Given the region feature vectors with 4 components, the feature vector for a transition corresponds to a point in the 8-dimensional spatial cooccurrence space. For simplicity, we refer to these points as $\mathbf{x}_{\boldsymbol{k}} \in \mathbb{R}^{d}, k=1, \ldots, N_{T}$ where $d=8$ and $N_{T}$ is the number of transitions.

We assume that the transitions that involve two similar region pairs fall close to each other in the spatial co-occurrence space because regions with similar spectral content and sizes are expected to be similar in terms of their features. Consequently, the transitions that occur frequently cause the accumulation of points in the space. While similar transitions are pooled together to form dense clusters, seldom transitions are located sparsely. This model provides tolerance to small variations and noise in the region features. Furthermore, it can easily be extended with additional region features.

The significance of a particular transition can be determined according to its location relative to the dense areas in the spatial co-occurrence space. We model this space with a Parzen window-based probability density estimate

$$
p(\mathbf{x})=\frac{1}{N_{T}} \sum_{k=1}^{N_{T}} \frac{1}{(2 \pi)^{d / 2}|\mathbf{H}|^{1 / 2}} e^{-\frac{1}{2}\left(\mathbf{x}-\mathbf{x}_{k}\right)^{T} \mathbf{H}^{-1}\left(\mathbf{x}-\mathbf{x}_{\boldsymbol{k}}\right)}
$$

using a Gaussian kernel with a smoothing parameter $\mathbf{H}=\sigma^{2} \mathbf{I}$ (also called the bandwidth). The bandwidth $\mathbf{H}$ for a given data set is obtained using a leave-one-out maximum likelihood estimation procedure [5]. The main advantage of the Parzen density estimate is that it does not require any assumption about the shape of the density function.

\subsection{Finding important relations}

We assume that the dense regions in this space correspond to the most frequently occurring and hence the most significant and important transitions. These dense regions can be found by locating the modes (local maxima) of the estimated density. We obtain these modes using the mean-shift algorithm [6]. Starting from a randomly selected set of points, the algo- 
rithm computes the mean-shift vector at each point $\mathbf{x}$ as

$$
\mathbf{m}(\mathbf{x})=\frac{\sum_{k=1}^{N_{T}} \mathbf{x}_{\boldsymbol{k}} e^{-\frac{1}{2}\left(\mathbf{x}-\mathbf{x}_{\boldsymbol{k}}\right)^{T} \mathbf{H}^{-1}\left(\mathbf{x}-\mathbf{x}_{\boldsymbol{k}}\right)}}{\sum_{k=1}^{N_{T}} e^{-\frac{1}{2}\left(\mathbf{x}-\mathbf{x}_{\boldsymbol{k}}\right)^{T} \mathbf{H}^{-1}\left(\mathbf{x}-\mathbf{x}_{\boldsymbol{k}}\right)}}-\mathbf{x}
$$

using the Parzen density gradient estimate at that point, and moves along this vector by iterating until the difference between two successive means is less than a convergence threshold or the number of iterations reaches a maximum value. The points at which the algorithm converges are considered as the candidate modes.

The convergence of the mean-shift algorithm is affected by the convergence threshold and the number of maximum iterations allowed. Due to local details in the spatial cooccurrence space, starting at points that actually belong to the same mode may result in convergence at slightly different locations. To eliminate such noisy convergence, we merge the candidate modes that are closer to each other than the bandwidth. Further elimination can be done due to the symmetric nature of the co-occurrence space. Since the transition $T_{i j}$ is equivalent to transition $T_{j i}$, we compare the corresponding parts of the feature vectors of the candidate modes, and eliminate one of such mode pairs corresponding to symmetric transitions. The resulting set of modes provide an implicit clustering of the spatial co-occurrence space as any point in this space can be assigned to its closest mode.

\section{FINDING COMPOUND STRUCTURES}

Once the important relations are discovered, this information is employed in the translation of the image segmentation to the relational graph structure. The details of graph construction and subgraph analysis for finding compound structures are described below.

\subsection{Graph construction}

A relational graph is constructed from the segmentation of the whole scene so that the nodes represent the regions and there is an edge between the nodes that correspond to the adjacent regions. In particular, for each region $R_{i}$ there is a corresponding vertex $R_{i}$, and for each transition $T_{i j}$ there is an edge connecting the nodes $R_{i}$ and $R_{j}$.

It is common to use an unweighted graph and let the edges represent only the spatial adjacency [7]. However, by using this approach we may lose the detailed contextual information and the results may also suffer from the errors in segmentation. As described in Section 3.2, we assume that the modes of the density estimate of the spatial co-occurrence space correspond to the most significant and important transitions. This information is reflected in the constructed graph edges. First, the candidate modes with a probability smaller than a threshold are eliminated as such modes are likely to correspond to noisy, rare or insignificant transitions in sparse regions of the co-occurrence space. Then, the graph edges corresponding to the transitions that belong to the eliminated modes are removed. Furthermore, the graph can also be extended so that it reflects the transition type information. The transitions that are assigned to the same mode are accepted as a relation of the same type, and each transition (and the corresponding edge) is assigned an integer label between 1 and $N_{M}$ (the number of selected modes). As a result, the relationship information is fully encoded in the graph edges and their labels.

\subsection{Subgraph analysis}

The final objective is to find compound structures that are comprised of the subgraphs of the complete scene graph. In this paper, we use a method that was introduced in [8] and was implemented in the Subdue system for graph-based knowledge discovery. In our case, the input to the system is an undirected graph with labeled edges (the nodes are not labeled as we do not perform any classification of the regions after segmentation). Subdue searches for substructures (subgraphs) of the input graph that best compress this graph. The compression of the graph by a subgraph is defined as the replacement of this subgraph by a single node in the graph. The compression ability of a subgraph during the search is computed by the minimum description length heuristic [8]

$$
\text { Compression }=\frac{D L(S)+D L(G \mid S)}{D L(G)}
$$

where $S$ is the subgraph being evaluated, $D L(S)$ is the description length of $S, D L(G \mid S)$ is the description length of the input graph $G$ after it has been compressed using $S$, and $D L(G)$ is the description length of $G$. The description length is computed in terms of the number of bits required to encode a graph. The best subgraph is the one that minimizes (3).

The search is performed iteratively by compressing the graph with the best subgraph found in each iteration. The output is a list of subgraphs (in terms of the nodes and the edges they contain) that represent the discovered patterns together with all occurrences of each subgraph in the input graph. These subgraph instances are expected to constitute parts of compound structures in the complex urban scene.

\section{EXPERIMENTS}

To illustrate the effectiveness of the proposed method, we performed proof-of-concept experiments on the multi-spectral Ikonos image shown in Figure 1(a). The third segmentation scale (Figure 1(b)) was chosen among the 11 scales produced by RHSEG. The 51,558 regions present in this scale resulted in 263,246 transitions forming the points in the spatial cooccurrence space. By using these data, the bandwidth parameter was estimated as $\sigma=0.0188$.

The convergence threshold for the mean-shift algorithm was empirically set to $10^{-6}$ and the maximum number of it- 


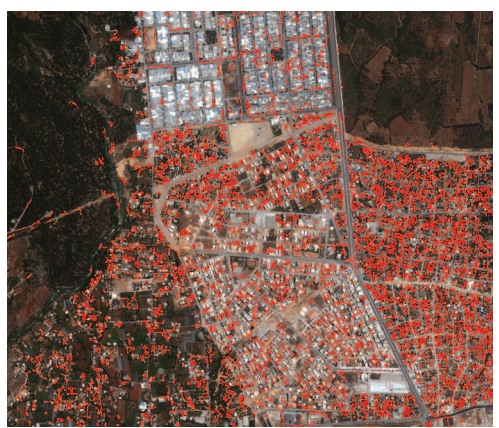

(a)

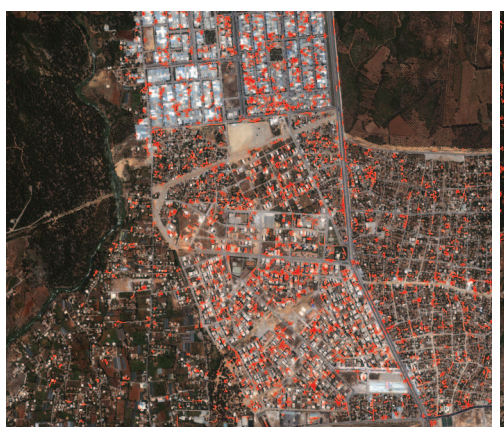

(b)



(c)

Fig. 2. Example substructures obtained by graph analysis. The regions that are involved in different substructure instances are shown in red in different subfigures.

erations allowed was 4,000. We ran the algorithm 1,400 times starting at different sets of randomly selected points. This resulted in 1,197 unique candidate modes. After mode merging and the elimination of the symmetric modes, the number of modes was reduced to 271 .

95 modes were chosen as significant $\left(N_{M}=95\right)$ by applying a threshold to the corresponding probability values. The Subdue algorithm was applied to the constructed graph, and the resulting substructures (subgraphs) were examined. Some example substructures and the corresponding region groups are shown in Figure 2. Even though a single substructure does not exclusively correspond to a particular compound structure, we can observe that different substructures constitute parts of different compound structures. For example, the substructure instances in Figure 2(a) mostly constitute the parts of residential areas with low height buildings. Similarly, the instances in 2(b) mainly correspond to parts of an industrial area and a residential area with high buildings, and the instances in 2(c) are contained within a forest.

We observed that the quality of the initial segmentation strongly influences the effectiveness of the following graph analysis. Future work includes improving the segmentation results and evaluating other graph clustering techniques for finding the interesting subgraphs.

\section{CONCLUSIONS}

Unlike the conventional object-based image analysis approach of finding homogeneous regions, we presented an unsupervised method toward discovering compound image structures that were comprised of complex groups of simpler primitive objects. We assumed that the primitive region objects that appeared together frequently could be considered as strongly related. Such potentially important relations were discovered using the modes of a probability distribution estimated using the features of the transitions between the neighboring regions in the image. The resulting modes were used to construct the edges of a graph in which the primitive regions form the nodes. A subgraph analysis algorithm was used to obtain the substructures of interest. Initial experiments on an Ikonos image showed that the algorithm has the potential for discovering different high-level compound structures in very high spatial resolution images.

\section{REFERENCES}

[1] S. Bhagavathy and B. S. Manjunath, "Modeling and detection of geospatial objects using texture motifs," IEEE Trans. on GRS, vol. 44, no. 12, pp. 3706-3715, 2006.

[2] E. Dogrusoz and S. Aksoy, "Modeling urban structures using graph-based spatial patterns," in IGARSS, 2007.

[3] M. Stasolla and P. Gamba, "Spatial indexes for the extraction of formal and informal human settlements from high-resolution SAR images," IEEE JSTARS, vol. 1, no. 2, pp. 98-106, 2008.

[4] J. C. Tilton, "Parallel implementation of the recursive approximation of an unsupervised hierarchical segmentation algorithm," in High-performance Computing in Remote Sensing, A. Plaza and C.-I. Chang, Eds. 2007.

[5] R. P. W. Duin, "On the choice of smoothing parameters for parzen estimators of probability density functions," IEEE Trans. on Computers, vol. C-25, no. 11, pp. 11751179, 1976.

[6] D. Comaniciu and P. Meer, "Mean shift: a robust approach toward feature space analysis," IEEE Trans. on PAMI, vol. 24, no. 5, pp. 603-619, 2002.

[7] J. C. Tilton, D. J. Cook, and N. Ketkar, "The integration of graph based knowledge discovery with image segmentation hierarchies for data analysis, data mining and knowledge discovery," in IGARSS, 2008.

[8] D. J. Cook and L. B. Holder, "Graph-based data mining," IEEE Intelligent Systems, vol. 15, no. 2, pp. 32-41, 2000. 